\title{
THE ATIYAH-SINGER INVARIANT, TORSION INVARIANTS, AND GROUP ACTIONS ON SPHERES
}

\author{
BY \\ DONALD E. SMITH ${ }^{1}$
}

\begin{abstract}
This paper deals with the classification of cyclic group actions on spheres using the Atiyah-Singer invariant and Reidemeister-type torsion. Our main tool is the computation of the group of relative homotopy triangulations of the product of a disk and a lens space. These results are applied to obtain lower bounds on the image of an equivariant $J$-homomorphism.
\end{abstract}

Introduction. Smooth actions of finite groups on spheres which are semifree (the only isotropy groups are the identity subgroup and the whole group) and semilinear (the fixed set is a homotopy sphere) have received a considerable amount of attention in the past decade (see $[\mathbf{2 0 , 2 1}$ ) and references there). Rothenberg [16] produced exact sequences relating groups of semilinear spheres to Wall groups and homotopy groups of classical groups and function spaces. Similar results were announced by Browder and Petrie [5] who carried out rational calculations using the Atiyah-Singer invariant (see the remarks after 5.4). Subsequently, Schultz refined and generalized these techniques to obtain much more detailed information $[18,19,20]$. Finally, Ewing's application of the $G$-signature theorem to semifree actions $[7,8]$ was an important contribution to the analysis of Rothenberg's sequences (see 3.4, 5.3 below).

In this paper, we study PL actions which are semifree and semilinear, as well as smooth near the fixed set. The advantage of this hybrid category is that in it explicit calculations with the Atiyah-Singer invariant can be carried out which allow classification of semilinear spheres. These are modeled on Wall's study of free piecewise linear actions of odd order cyclic groups on spheres $[25, \S 14 \mathrm{E}]$. Out of these calculations come the invariants which detect elements in the image of an equivariant $J$-homomorphism.

1. Definitions and summary of results. Let $G$ be a cyclic group of odd order $q$ and let $\alpha=n \oplus u$ be a representation of $G$ which is the sum of the trivial $n$ dimensional real representation and an orthogonal representation $u$ such that $G$ acts freely on $S(u)$, the unit sphere of $u$. An $\alpha$-manifold is a PL manifold with a semifree PL $G$-action together with the following additional structure: (1) A neighborhood of the fixed set is provided with a sliced concordance class of $G$-smoothings [11]. (2) The

Received by the editors June 3, 1981.

1980 Mathematics Subject Classification. Primary 57S25; Secondary 57R67, 55Q50.

'This work was partially supported by NSF Grant MCS 77-18723 A04.

(C)1983 American Mathematical Society 0002-9947/82/0000-0717/\$06.00 
local representation of $G$ at a fixed point is equivalent to $\alpha$. The appropriate notions of PL homeomorphism, cobordism, etc. are those which preserve this additional structure.

Let $S_{\alpha}$ be the set of $G$-PL homeomorphism classes of semilinear $\alpha$-spheres. Let $C S_{\alpha}$ be the set of $G h$-cobordism classes of semilinear $\alpha$-spheres. Lastly, $R S_{\alpha}$ will consist of pairs $(\Sigma, t)$ where $\Sigma$ is a semilinear $\alpha$-sphere whose fixed set $F$ is diffeomorphic to the standard sphere $S^{n}$ and $t: S^{n} \times D(u) \rightarrow \Sigma$ is an equivariant trivialization of the normal bundle of $F$ in $\Sigma$. On such pairs we put the equivalence relation of $G$ $h$-cobordism preserving the trivialization. As in the smooth case $[16,18]$, equivariant orientations give these sets the structures of abelian groups via connected sum.

The group $S_{\alpha}$ is what we would like to compute. In $\S 2$, we use torsion invariants to reduce its computation to that of $C S_{\alpha}$. There are exact sequences just like those in [16] relating $C S_{\alpha}$ to $R S_{\alpha}$ and $R S_{\alpha}$ to more familiar groups. Moreover, as explained in $\S 3$, the Atiyah-Singer invariant is defined on $R S_{\alpha}$, so it is a fairly tractable group. In $\S 3$, we also analyze the dependence of the Atiyah-Singer invariant on the trivialization.

The primary method of constructing elements of $R S_{\alpha}$ uses $S_{\mathrm{PL}}^{h}\left(D^{n+1} \times L, S^{n} \times L\right)$ the group of relative homotopy triangulations $(L=S(u) / G)$ : To the universal cover of a homotopy $D^{n+1} \times L$ one glues $S^{n} \times D(u)$ equivariantly to get an $\alpha$-sphere. This group is the subject of $\S 4$. It is calculated precisely using the Atiyah-Singer invariant, Whitehead torsion, and a so-called splitting invariant.

In $§ 5$, we use the results of the preceding sections to study $C S_{\alpha}$ and find that it finite if $n$ is even and described by our invariants modulo the cokernel of an equivariant $J$-homomorphism if $n$ is odd. Then we turn to the image of this homomorphism. The classification results of $\$ 4$ imply integrality conditions for our invariants which enable us to obtain lower bounds on the order of the image of the equivariant $J$-homomorphism much in the way that integrality conditions on the $\hat{A}$-genus enabled Kervaire and Milnor [10] to estimate the image of the classical $J$-homomorphism. Precisely, let $F(u)$ be the space of self $G$-maps of $S(u)$ and let $C(u)=F(u) \cap O(2 d), 2 d=\operatorname{dim} u$. Our equivariant $J$-homomorphism

$$
J(u): \pi_{4 k-1} C(u) \rightarrow \pi_{4 k-1} F(u)
$$

is induced by the inclusion $C(u) \rightarrow F(u)$. Forgetting the $G$-action gives $\varphi$ : image $J(u) \rightarrow$ image $J$ where $J: \pi_{4 k-1} S O \rightarrow \pi_{4 k-1}^{S}$ is the stable $J$-homomorphism.

THEOREM 5.9. Let $u$ underlie the unitary representation of $\mathbf{Z}_{q}$ taking 1 to the $d \times d$ diagonal matrix with entries $\lambda=e^{2 \pi i / q}$ where $d>2 k$. Suppose $q>2 k+1$ is an odd prime. Then the Atiyah-Singer invariant induces a homomorphism

$$
\pi_{4 k-1} F(u) \rightarrow \mathbf{Q}(\lambda) / \mathbf{Z}[\lambda]
$$

which detects a subgroup of order $q$ in the kernel of $\varphi$.

Since the cokernel of $\varphi$ is easily determined, $\operatorname{ker} \varphi$ is the only unknown part of image $J(u)$.

Some of the results of this paper are contained in my $\mathrm{Ph} . \mathrm{D}$. dissertation written at the University of Chicago under the direction of Professor M. Rothenberg. I would 
like to acknowledge the National Science Foundation's financial support of this research.

2. A torsion invariant. In this section, we define a torsion invariant for semifree actions on disks and spheres and use it to reduce the equivariant PL homeomorphism classification problem to the problem of equivariant $h$ cobordism classification. This invariant is based on Milnor's torsion invariant for free actions $[13,15]$. The results of this section hold for smooth actions as well as our PL actions.

Let $X$ be a semifree finite $G-\mathrm{CW}$ complex. Let $C$ denote the cellular chain complex $C_{*}\left(X, X^{G} ; \mathbf{Q}\right)$. It is free over the rational group ring $\mathbf{Q} G$. Suppose that $G$ acts trivially on the homology of $C$. Set $\mathbf{Q} R_{G}=\mathbf{Q} G / \mathbf{Q} \Sigma$ where $\Sigma$ is the sum of all the elements of $G$. Then $\mathbf{Q} R_{G} C$ is a free, acyclic $\mathbf{Q} R_{G}$-complex and has a basis corresponding to the cells of $\left(X-X^{G}\right) / G$. So it has a well-defined torsion.

Definition 2.1. The torsion of $X$, denoted by $\tau(X)$, is

$$
\tau\left(\mathbf{Q} R_{G} C\right) \in K_{1}\left(\mathbf{Q} R_{G}\right) / \pm G .
$$

If $X$ is a semilinear sphere or disk, $\tau(X)$ is defined.

Definition 2.2. Let $X \in S_{\alpha}$. Then $\tau_{\alpha}(X)=\tau(S(\alpha \oplus 1))-\tau(X)$.

The reason for introducing $\tau_{\alpha}$ is that it is a homomorphism

$$
\tau_{\alpha}: S_{\alpha} \rightarrow K_{1}\left(\mathbf{Q} R_{G}\right) / \pm G
$$

as one checks using excision. The following theorem is the basis of most results about $\tau_{\alpha}$.

THEOREM 2.3 [17]. Let $D_{\alpha}$ be the group of $\alpha$-disks and $D S_{\alpha}$ the group of equivariant PL homeomorphisms of $S(\alpha)$ (under a suitable relation; see [17]). Then there is an exact sequence

$$
0 \rightarrow D S_{\alpha} \rightarrow S_{\alpha \oplus 1} \stackrel{k_{\alpha \oplus 1}}{\rightarrow} D_{\alpha \oplus 1} \stackrel{j_{\alpha \oplus 1}}{\rightarrow} S_{\alpha} \rightarrow C S_{\alpha} \rightarrow 0
$$

where the first map glues two copies of $D(\alpha)$ together, $k$ deletes a linear disk, $j$ takes the boundary, and the last map is obvious. Moreover, if $\operatorname{dim} u>2$ and $n>1$ there is an isomorphism $t_{0}: D_{\alpha} \rightarrow \mathrm{Wh} G$ and the composite

$$
\mathrm{Wh} G \approx D_{\alpha \oplus 1} \stackrel{k_{\alpha} j_{\alpha \oplus 1}}{\rightarrow} D_{\alpha} \approx \mathrm{Wh} G
$$

is multiplication by $1+(-1)^{n+1}$.

Proposition 2.4. There is a commutative diagram

$$
\begin{array}{ccc}
S_{\alpha} & \stackrel{k}{\rightarrow} & D_{\alpha} \\
\downarrow \tau_{\alpha} & & \downarrow t_{0} \\
K_{1}\left(\mathbf{Q} R_{G}\right) / \pm G & \stackrel{-p}{\leftarrow} & \mathrm{Wh} G
\end{array}
$$

where $p$ is induced by $\mathbf{Z} G \rightarrow \mathbf{Q} G$. 
Proof. If $X \in S_{\alpha}$, regard $k(X)$ as a subset of $X$. There is a based exact sequence

$$
0 \rightarrow C_{*}\left(k(X), k\left(X^{G}\right)\right) \rightarrow C_{*}\left(X, X^{G}\right) \rightarrow C_{*}\left(X, X^{G} \cup k(X)\right) \rightarrow 0
$$

and a relative $G$-cellular isomorphism

$$
C_{*}\left(X, X^{G} \cup k(X)\right) \approx C_{*}\left(S(\alpha \oplus 1), S(\alpha \oplus 1)^{G}\right) .
$$

Thus, $\tau(X)=\tau(S(\alpha \oplus 1))+p(\tau(k(X)))$. This completes the proof.

Hence $\tau_{\alpha}$ factors through the Whitehead group. In fact, it is well known that $p$ is injective, so we shall henceforth regard $\tau_{\alpha}$ as a homomorphism $\tau_{\alpha}: S_{\alpha} \rightarrow$ Wh $G$.

Proposition 2.5. Let $\alpha=n \oplus u$, where $n>1$ is odd and $2 d=\operatorname{dim} u>2$. Then $\tau_{\alpha}$ is surjective, while $\tau_{\alpha \oplus 1}$ is zero.

Proof. By 2.3 and 2.4, we have a commutative diagram

$$
\begin{aligned}
& 0 \rightarrow D_{\alpha \oplus 1} \stackrel{j}{\rightarrow} \quad S_{\alpha} \quad \rightarrow \quad C S_{\alpha} \quad \rightarrow \quad 0 \\
& \approx \downarrow \quad \downarrow \tau_{\alpha} \quad \downarrow \tau_{\alpha}^{\prime} \\
& 0 \rightarrow \mathrm{Wh} G \stackrel{-2}{\rightarrow} \mathrm{Wh} G \rightarrow \mathrm{Wh} G / 2 \mathrm{Wh} G \rightarrow 0
\end{aligned}
$$

so it will suffice to prove that $\tau_{\alpha}^{\prime}$ is onto. For this, consider the diagram:

$$
\begin{array}{ccccc}
L_{n+2 d+1}^{s}(G) & \rightarrow & L_{n+2 d+1}^{h}(G) & & \stackrel{D}{\rightarrow} \mathrm{Wh} G / 2 \mathrm{Wh} G \rightarrow 0 \\
\downarrow & & & \| \\
S_{\mathrm{PL}}^{s}\left(\left(D^{n+1}, S^{n}\right) \times L\right) & \rightarrow & S_{\mathrm{PL}}^{h}\left(\left(D^{n+1}, S^{n}\right) \times L\right) & \stackrel{\tau^{\prime}}{\rightarrow} \mathrm{Wh} G / 2 \mathrm{Wh} G \rightarrow 0
\end{array}
$$

The first row is the Rothenberg sequence relating Wall groups for simple and ordinary homotopy equivalences [22]. $D$ is onto since $L_{\text {odd }}(G)=0$ by [4]. Hence $\tau^{\prime}$, the reduced Whitehead torsion, is onto. If $g^{\prime}$ is the composite $S_{\mathrm{PL}}^{h} \stackrel{g}{\rightarrow} R S_{\alpha} \rightarrow C S_{\alpha}$, where $g$ is as in $\S 4$ and the second map is obvious, then since $\tau_{\alpha}^{\prime} g^{\prime}=\tau^{\prime}$, it follows that $\tau_{\alpha}$ is onto.

To see that $\tau_{\alpha \oplus 1}=0$, we simply note that $S_{\alpha \oplus 1} \rightarrow D_{\alpha \oplus 1}$ is zero since $\tau_{\alpha} \circ j \circ t_{0}^{-1}$ is multiplication by -2 . But $-\tau_{\alpha \oplus 1}$ is the composite $S_{\alpha \oplus 1} \rightarrow D_{\alpha \oplus 1} \approx$ Wh $G$ by 2.4 .

REMARK. The sequence relating $S^{s}$ and $S^{h}$ in the preceding proof is actually short exact.

3. The Atiyah-Singer invariant. In this section, we discuss the Atiyah-Singer invariant and define it on $R S_{\alpha}$. We then investigate the influence of the trivialization of the fixed set's normal tube on the invariant.

Let $M^{2 k-1}$ be a closed, oriented PL manifold with a free $G$ action. Then a number (say $r$ ) of copies of $M$ is the oriented boundary of an oriented, free $G$-manifold $W^{2 k}$. Let $\operatorname{Sign}(G, W)$ be the $G$-signature of $W$ and $\operatorname{Sign}(g, W)$ its value at $g \in G$.

Definition 3.1. The Atiyah-Singer invariant of $M$ is the function on $G-\{1\}$ with values in $\mathbf{Q}\left(e^{2 \pi i / q}\right)$ given by

$$
\sigma(g, M)=-\frac{1}{r} \operatorname{Sign}(g, W) .
$$


The restriction to $G-\{1\}$ is made so that $\sigma(M)$ is independent of $W . \sigma$ is real-valued if $k$ is even and imaginary if $k$ is odd. It depends only on the equivariant $h$-cobordism class of $M$.

A very useful generalization of 3.1 for smooth manifolds is obtained if $W$ is not required to have a free action. In this case,

$$
\sigma(g, M)=r^{-1}(L(g, W)-\operatorname{Sign}(g, W))
$$

where $L(g, W)$ is the characteristic number in the $G$-signature theorem $[3,6.12,7.4]$.

For our purposes, a reformulation of $\sigma$ will be convenient. Let $T$ be a preferred generator of $G$ and let $\hat{G}$ be the dual group of $G$. It is cyclic of order $q$ and has as preferred generator the character $\chi$ taking $T$ to $e^{2 \pi i / q}$. Regard the $G$-signature as a virtual representation, i.e., as an element of $\mathbf{Z} \hat{G}$. Restricting to $G-\{1\}$ means factoring out by the regular representation $1+\chi+\chi^{2}+\cdots+\chi^{q-1}$. Division by $r$ then puts $\boldsymbol{\sigma}$ in $\mathbf{Q} R_{\hat{G}}=\mathbf{Q} \otimes R_{\hat{G}}$, where $R_{\hat{G}}=\mathbf{Z} \hat{G} / \mathbf{Z}\left(1+\chi+\cdots+\chi^{q-1}\right)$. Actually, $\sigma$ lies in $R_{\hat{G}}[1 / q]$ as can be proved by a bordism spectral sequence argument.

Now let $(\Sigma, t)$ represent an element of $R S_{\alpha}$, where $\alpha=(2 m-1) \oplus u$. Set

$$
\Sigma_{t}=\left(\Sigma-t\left(S^{2 m-1} \times \text { interior } D(u)\right)\right) \cup_{\partial t} D^{2 m} \times S(u),
$$

the result of surgery on $t$. We then define $\sigma(\Sigma, t)=\sigma\left(\Sigma_{t}\right)$.

LEMMA 3.2. The Atiyah-Singer invariant induces

$$
\sigma: R S_{\alpha} \rightarrow R_{\hat{G}}[1 / q], \quad \alpha=(2 m-1) \oplus u,
$$

which is a homomorphism if $m \neq d(2 d=\operatorname{dim} u)$.

The proof is a routine but complicated application of Wall's generalized Novikov additivity theorem [24].

Now we shall study the dependence of the Atiyah-Singer invariant on the trivialization of the normal bundle of the fixed set in the ambient sphere. If $C(u)$ is the centralizer of $u(T)$ in $O(2 d)$, then $G$-trivializations of $S^{n} \times D(u)$ correspond to maps $h: S^{n} \rightarrow C(u)$. We obtain a homomorphism $f: \pi_{n} C(u) \rightarrow R S_{\alpha}$ by taking $h$ to the standard $\alpha$-sphere $S(\alpha \oplus 1)$ with

$$
S^{n} \times D(u) \stackrel{\hat{h}}{\rightarrow} S^{n} \times D(u) \subset S(\alpha \oplus 1)
$$

as trivialization, where $\hat{h}(x, y)=(x, h(x)(y))$. When $n=2 m-1$ is odd, we can use $\sigma$ to analyze $f$.

First, we need to specify our representation $u$ which, so far, has figured only as an equivalence class. Henceforth $u$ will be the real representation underlying the unitary representation of complex dimension $d$ taking the preferred generator $T$ of $G$ to the diagonal matrix with entries of the form $\lambda^{k}$, where $\lambda=e^{2 \pi i / q}$ and $k \in A=\{j \in \mathbf{Z} \mid$ $(j, q)=1$ and $1 \leqslant j<q / 2\}$. If $\lambda^{k}$ has multiplicity $d_{k}$, then $C(u)$ is isomorphic to the product of unitary groups $\prod_{k \in A} U\left(d_{k}\right)$. Since the structure group of a $G$-vector bundle $\xi$ with fiber $u$ over a trivial $G$-space reduces to $C(u)[6, \S 35]$, $\xi$ splits as a direct sum of complex $d_{k}$ dimensional bundles $\xi_{i}, k \in A$. 
Now let $\xi \in \pi_{2 m-1} C(u)$ (we use $\xi$ to denote the corresponding $G$ vector bundle over $S^{2 m}$ also). If $(\Sigma, t)=f(\xi)$, then $\Sigma_{t}$ is the sphere bundle $S(\xi)$ of $\xi$. Since $S(\xi)$ bounds the associated disk bundle $D(\xi)$,

$$
\sigma(T, f(\xi))=\sigma(T, S(\xi))=L(T, D(\xi))-\operatorname{Sign}(T, D(\xi)) .
$$

$\operatorname{Sign}(G, D(\xi))$ is the $G$-signature of a bilinear form on the cohomology of $(D(\xi), S(\xi))$ (see [3, p. 588]) and is zero if $m \neq d$. To describe $L(T, D(\xi)$ ), we recall from [2, Theorem 6.27] that the Atiyah-Singer invariant of $S(u)$, which we shall denote by $\sigma(L)(L=S(u) / G)$, is given by

$$
\sigma(L)=\sigma(G, S(u))=\prod_{k \in A}\left(\frac{\chi^{k}+1}{\chi^{k}-1}\right)^{d_{k}}
$$

as an element of $\mathbf{Q} R_{\hat{G}}$. Following [8], let $\Re\left(\lambda^{k}, \xi_{k}\right)$ be the multiplicative sequence belonging to the power series

$$
\left(\frac{\lambda^{k}-1}{\lambda^{k}+1}\right)\left(\frac{\lambda^{k} e^{t}+1}{\lambda^{k} e^{t}-1}\right)
$$

Then $L(T, D(\xi))=2^{m} \sigma(T, L) \Pi_{k \in A} \Re\left(\lambda^{k}, \xi_{k}\right)\left[S^{2 m}\right]$. As in [8], $\Re\left(\lambda^{k}, \xi_{k}\right)=1+$ $\Phi_{m}\left(\lambda^{k}\right) c_{m}\left(\xi_{k}\right)$, where $\Phi_{m}\left(\lambda^{k}\right)$ is a polynomial expression in $\lambda^{k}$ with rational coefficients and $c_{m}$ is the $m$ th Chern class. Thus

$$
L(T, D(\xi))=2^{m} \sigma(T, L) \sum_{k \in A} \Phi_{m}\left(\lambda^{k}\right) c_{m}\left(\xi_{k}\right)\left[S^{2 m}\right] .
$$

A Chern number in the sum is zero unless $d_{k} \geqslant m$ since $\pi_{2 m-1} U\left(d_{k}\right)$ is finite if $d_{k}<m$ and isomorphic to $\mathbf{Z}$ if $d_{k} \geqslant m$. According to [8, Lemma 2.4], the numbers $\Phi_{m}\left(\lambda^{k}\right), k \in A$, are linearly independent over the rationals if $m>1$.

Proposition 3.4. Assume $m>1$ and $m \neq d$. The homomorphism $\pi_{2 m-1} C(u) \rightarrow$ $\mathbf{Q}(\lambda)$ given by $\xi \mapsto \sigma(T, f(\xi))$ induces an injection on $\pi_{2 m-1} C(u) /$ Torsion. Thus $\pi_{2 m-1} C(u) /$ Torsion $\rightarrow R S_{\alpha}$ is injective.

REMARK. The injectivity statements are valid if $m=d$, too.

For our work below on the equivariant $J$-homomorphism, we need some information about the polynomials $\Phi_{m}\left(\chi^{k}\right)$ in $\chi^{k}$. Since $\Phi_{m}\left(\chi^{k}\right)$ is obtained from $\Phi_{m}(\chi)$ by replacing $\chi$ with $\chi^{k}$, it suffices to study $\Phi_{m}(\chi)$. We begin with the identity [8, p. 436]

$$
\sum_{m=1}^{\infty} \Phi_{m}(\chi)(-t)^{m}=-t \frac{d}{d t} \log \left(\frac{\chi-1}{\chi+1} \frac{\chi e^{t}+1}{\chi e^{t}-1}\right)=\frac{2 \chi t e^{t}}{\chi^{2} e^{2 t}-1}
$$

in the formal power series ring $\mathbf{Q} \hat{G}[[t]]$. Letting $f(t)=2 \chi t e^{t} /\left(\chi^{2} e^{2 t}-1\right)$, we have $\Phi_{m}(\chi)=(-1)^{m} f^{(m)}(0) / m$ !. By differentiating $f$ repeatedly and using induction, one may prove

LEMMA 3.5. We may write

$$
\Phi_{m}(\chi)=\frac{2 \chi Q_{m}\left(\chi^{2}\right)}{(m-1) !\left(1-\chi^{2}\right)^{m}}
$$


where $Q_{m}$ is a polynomial of degree $\leqslant m-1$ with integer coefficients. Moreover, there is another polynomial $R_{m}$ with integer coefficients such that

$$
Q_{m}(y)=(-2)^{m-1}(m-1) ! y^{m-1}+(y-1) R_{m}(y) .
$$

For our final version of $\sigma(G, f(\xi))$, let $c_{m}^{\prime}\left(\xi_{k}\right)$ be given by

$$
c_{m}\left(\xi_{k}\right)\left[S^{2 m}\right]=(m-1) ! c_{m}^{\prime}\left(\xi_{k}\right) .
$$

This is possible by [9, p. 280].

Corollary 3.6. Suppose $m \neq d$. Let $\xi \in \pi_{2 m-1} C(u)$. Then

$$
\sigma(f(\xi))=2^{m+1} \sigma(L) \sum_{k \in A} \chi^{k}\left(1+\chi^{k}\right)^{-m} Q_{m}\left(\chi^{2 k}\right) c_{m}^{\prime}\left(\xi_{k}\right)\left(1-\chi^{k}\right)^{-m} .
$$

Proof. From 3.3, we have in $R_{\hat{G}}[1 / q]$

$$
L(G, D(\xi))=2^{m} \sigma(L) \sum_{k \in A} \Phi_{m}\left(\chi^{k}\right) c_{m}\left(\xi_{k}\right)\left[S^{2 m}\right] .
$$

Since $m \neq d, \sigma(f(\xi))=L(G, D(\xi))$. The desired formula now follows from 3.5 and the definition of $c_{m}^{\prime}$.

4. Homotopy triangulations of $D^{n+1} \times L$. Let $S_{\mathrm{PL}}^{h}\left(D^{n+1} \times L, S^{n} \times L\right)$ be the abelian group of relative homotopy triangulations of $D^{n+1} \times L$, where $L=S(u) / G$ is the lens space of $u$. Given a homotopy equivalence $h: N \rightarrow D^{n+1} \times L$ such that $\partial h$ is a PL homeomorphism, let $\Sigma=\tilde{N} \cup_{\partial \tilde{h}} S^{n} \times D(u)$ where ${ }^{\sim}$ denotes universal cover. Give $S^{n} \times D(u)$ its natural trivialization $t_{0}$; then $\left(\Sigma, t_{0}\right)$ represents an element of $R S_{\alpha}$. Sending $h$ to $\left(\Sigma, t_{0}\right)$ induces a homomorphism

$$
g: S_{\mathrm{PL}}^{h}\left(D^{n+1} \times L, S^{n} \times L\right) \rightarrow R S_{\alpha}
$$

which is the most useful means of constructing elements of $R S_{\alpha}$.

This section is devoted to computing $S_{\mathrm{PL}}^{h}\left(D^{n+1} \times L, S^{n} \times L\right)$ and is based on C. T. C. Wall's classification of fake lens spaces $[25, \S 14 \mathrm{E}]$. The answer is complete when $u$ is the representation $d \chi$ with $d \chi(T)=\lambda \cdot(d \times d$ identity matrix $)$ and nearly so in other cases. We use the surgery exact sequence to carry out the computation. Normal maps into $D^{n+1} \times L$ which are PL homeomorphisms on the boundary are classified by

$$
\left[\left(D^{n+1}, S^{n}\right) \times L ; F / \mathrm{PL}\right] \approx \pi_{n+1} F / \mathrm{PL} \oplus\left[\Sigma^{n+1} L, F / \mathrm{PL}\right] .
$$

(Here, [ ] denotes based homotopy classes of based maps; spaces without natural base points are given arbitrary base points.) First we introduce a suspension construction for triangulations which enables us to construct and classify all normal maps. Then we use the $G$-signature on Wall groups to complete the calculation.

LEMMA 4.1. Let $c: L^{2 d-1} \rightarrow S^{2 d-1}$ collapse the $(2 d-2)$-skeleton of $L$ to a point. Then

$$
c^{*}: \pi_{2 m+2 d} F / \mathrm{PL} \rightarrow\left[\Sigma^{2 m+1} L^{2 d-1}, F / \mathrm{PL}\right]
$$

is an isomorphism. The order of $\left[\Sigma^{2 m} L^{2 d-1}, F / \mathrm{PL}\right]$ is $q^{[d / 2]}$ if $m$ is odd and $q^{[(d-1) / 2]}$ if $m$ is even $([r]$ is the greatest integer $\leqslant r)$. 
Proof. Let $\pi: S^{2 d-1} \rightarrow L$ be the covering projection. The three maps $\pi, c$, and $c \pi$ have cofibration sequences which fit into a braid diagram [12, p. I.10]. Applying the functor $[-, F / \mathrm{PL}]$ gives a braid of exact sequences. Since $\pi_{*} F / \mathrm{PL}$ is known, we can use inductive arguments to extract the statements of the lemma from this braid.

Corollary 4.2. For any integer $m \geqslant 0, S_{\mathrm{PL}}^{c}\left(D^{2 m+1} \times L, S^{2 m} \times L\right)=0$ where $c=h$ (homotopy) or s (simple homotopy).

Proof. In the surgery exact sequence

$$
\begin{aligned}
L_{2 m+2 d+1}^{c}(G) & \rightarrow S_{\mathrm{PL}}^{c}\left(D^{2 m+1} \times L, S^{2 m} \times L\right) \\
& \rightarrow \pi_{2 m+1} F / \mathrm{PL} \oplus\left[\Sigma^{2 m+1} L, F / \mathrm{PL}\right] \stackrel{\theta}{\rightarrow} L_{2 m+2 d}^{c}(G),
\end{aligned}
$$

$L_{2 m+2 d+1}^{c}(G)=0$ by [4]. Since $\pi_{2 m+1} F / \mathrm{PL}=0$ and $\theta$ is injective by the diagram

$$
\begin{array}{ccc}
{\left[\Sigma^{2 m+1} L, F / \mathrm{PL}\right]} & \stackrel{\theta}{\rightarrow} & L_{2 m+2 d}^{c}(G) \\
\uparrow c^{*} & & \uparrow \text { injective } \\
\pi_{2 m+2 d} F / \mathrm{PL} & \stackrel{\sim}{\rightarrow} & L_{2 m+2 d}(1)
\end{array}
$$

and 4.1, the corollary follows.

We now introduce suspension. Let $\Sigma$ be an $\alpha$-sphere and give $S^{1}$ a free $G$-action via the character $\chi^{p}$ where $(p, q)=1$. Let $C \Sigma$ be the cone on $\Sigma$.

Definition 4.3. The suspension of $\Sigma$ is

$$
\Sigma * S^{1}=\Sigma \times D^{2} \cup C \Sigma \times S^{1},
$$

the join of $\Sigma$ and $S^{1}$ with diagonal $G$-action. If $t: S^{n} \times D(u) \rightarrow \Sigma$ is an equivariant trivialization of the normal bundle of $\Sigma^{G}$ in $\Sigma, t \times \operatorname{Id}_{D^{2}}: S^{n} \times D(u) \times D^{2} \rightarrow \Sigma \times$ $D^{2} \subset \Sigma * S^{1}$ trivializes the normal bundle of $\left(\Sigma * S^{1}\right)^{G}$ in $\Sigma * S^{1}$. We let $(\Sigma, t) * S^{1}$ denote the suspension of $\Sigma$ with this trivialization.

It is straightforward but tedious to prove that suspension is additive and compatible with respect to $h$-cobordisms. So we obtain, for example, a homomorphism $* S^{1}$ : $R S_{\alpha} \rightarrow R S_{\alpha \oplus \chi^{p}}$

LEMMA 4.4. If $\Sigma$ is an $\alpha=n \oplus u$-sphere, then

$$
\tau_{\alpha \oplus \chi^{p}}\left(\Sigma * S^{1}\right)=\tau_{\alpha}(\Sigma) \text {. }
$$

Proof. We show that $\tau\left(\Sigma * S^{1}\right)=\tau(\Sigma)-(-1)^{n} \tau\left(S^{1}\right)$. The lemma follows from this and the definition of $\tau_{\alpha}$. There is a relative $G$-cellular isomorphism $\left(\Sigma * S^{1}, \Sigma \cup\right.$ $\left.S^{1}\right) \rightarrow\left(S^{0} *\left(\Sigma \times S^{1}\right), S^{0}\right)$ (compare [15, proof of 4.1]). Since $\tau\left(S^{0} *\left(\Sigma \times S^{1}\right) / S^{0}\right)$ $=-\tau\left(\Sigma \times S^{1}\right), \tau\left(\Sigma * S^{1}\right)=\tau(\Sigma)+\tau\left(S^{1}\right)-\tau\left(\Sigma \times S^{1}\right)$. By [15, 1.28a], $\tau\left(\Sigma \times S^{1}\right)$ $=\left(1+(-1)^{n}\right) \tau\left(S^{1}\right)$, so $\tau\left(\Sigma * S^{1}\right)=\tau(\Sigma)-(-1)^{n} \tau\left(S^{1}\right)$.

THEOREM 4.5. Let $(\Sigma, t)$ represent an element of $R S_{\alpha}$. Then

$$
\sigma\left((\Sigma, t) * S^{1}\right)=\sigma(\Sigma, t) \sigma\left(S^{1}\right) .
$$

Proof. This is a generalization of [25, 14A.1]. Let $N$ be a free oriented $G$-manifold with $\partial N=r \cdot \Sigma_{t}$. Add handles $D^{n+1} \times D(u)$ along $D^{n+1} \times S(u)$ to each copy of $\Sigma_{t}$ 
in $\partial N$ to get a manifold with boundary $r \cdot \Sigma$. Cone off each copy of $\Sigma$ to get a closed manifold $\bar{N}$ whose $G$ signature equals that of $N$. Note that as oriented manifolds $\Sigma=\partial(-C \Sigma)$.

Let $P^{2}$ be a free oriented $G$-manifold whose boundary consists of $s$ copies of $S^{1}$. Cone off each to get a closed manifold $\bar{P}$. Note that $S^{1}=\partial\left(-C S^{1}\right)$.

The product $\bar{N} \times \bar{P}$ has a decomposition like that of $\bar{N}$. Namely, $\bar{N} \times \bar{P}$ consists of $r s$ copies of cones $C \Sigma \times C S^{1}$ glued to $D^{n+1} \times D(u) \times C S^{1}$ along $S^{n} \times$ $\partial\left(D(u) \times C S^{1}\right)$ together with a free $G$-manifold $W$. Now

$$
\partial\left(C \Sigma \times C S^{1}\right)=\partial\left(-C \Sigma \times-C S^{1}\right)=\Sigma * S^{1} .
$$

It follows that $\partial W=-r s\left(\Sigma * S^{1}\right)_{t \times \operatorname{Id}_{D^{2}}}$. Therefore

$$
\begin{aligned}
r s \sigma\left((\Sigma, t) * S^{1}\right) & =\operatorname{Sign}(G, W)=\operatorname{Sign}(G, N) \cdot \operatorname{Sign}(G, P) \\
& =(-r \sigma(\Sigma, t))\left(-s \sigma\left(S^{1}\right)\right)=r s \sigma(\Sigma, t) \sigma\left(S^{1}\right) .
\end{aligned}
$$

REMARK. There is an error in [25, 14A.1]. Wall does not take account of the fact that the orientation of $W$ coming from the product orientation on $\bar{N} \times \bar{P}$ induces the negative of the join orientation on the boundary. Thus his invariant $\rho=-\sigma$ is not multiplicative for joins; $\sigma$ is.

Suspension on $R S_{\alpha}$ induces a suspension on $S_{\mathrm{PL}}^{h}\left(D^{n+1} \times L, S^{n} \times L\right)$ as follows. Given $h: N \rightarrow D^{n+1} \times L, g(h)=\tilde{N} \cup S^{n} \times D(u)$ is an $\alpha$-sphere. If from $g(h) * S^{1}$ we delete $S^{n} \times D(u) \times C S^{1}$ and pass to quotients by $G$, we obtain a homotopy triangulation of $D^{n+1} \times L^{2 d+1}$, where $L^{2 d+1}=\left(S(u) * S^{1}\right) / G$. Thus we have a homomorphism

$$
* S^{1}: S_{\mathrm{PL}}^{c}\left(\left(D^{n+1}, S^{n}\right) \times L^{2 d-1}\right) \rightarrow S_{\mathrm{PL}}^{c}\left(\left(D^{n+1}, S^{n}\right) \times L^{2 d+1}\right) .
$$

We shall compute $S_{\mathrm{PL}}^{c}\left(\left(D^{2 m}, S^{2 m-1}\right) \times L^{2 d-1}\right)$ by applying three invariants to the modified surgery exact sequence

$$
\begin{aligned}
0 & \rightarrow \tilde{L}_{2 m+2 d}^{c}(G) \rightarrow S_{\mathrm{PL}}^{c}\left(\left(D^{2 m}, S^{2 m-1}\right) \times L\right) \\
& \rightarrow \pi_{2 m} F / \mathrm{PL} \oplus\left[\Sigma^{2 m} L, F / \mathrm{PL}\right] \rightarrow 0 .
\end{aligned}
$$

The first invariant is $\tau^{\prime}$, the mod 2 reduction of Whitehead torsion. The next is the Atiyah-Singer invariant, defined on $S_{\mathrm{PL}}^{c}$ by $\sigma(h)=\sigma(g(h))$. Finally,

$$
s_{0}: S_{\mathrm{PL}}^{c}\left(\left(D^{2 m}, S^{2 m-1}\right) \times L\right) \rightarrow \begin{cases}\mathbf{Z}, & m \text { even, } \\ \mathbf{Z}_{2}, & m \text { odd, }\end{cases}
$$

is called a splitting invariant. $s_{0}(h)$ is the surgery obstruction of the normal map obtained by making $h$ transverse to $D^{2 m} \times$ point. Both $s_{0}$ and $\tau^{\prime}$ are unaltered by suspension (the latter by 4.4$)$ and $\sigma\left(h * S^{1}\right)=\sigma(h) \sigma\left(S^{1}\right)$ by 4.5 .

Before our next result, we recall a fact about the behavior of $\sigma$ on the image of the Wall group and some facts about the ring $R_{\hat{G}}$ from [25, p. 205].

Proposition $4.7[25,13 \mathrm{~A} .4]$. Write $L_{2 n}(G)=L_{2 n}(1) \oplus \tilde{L}_{2 n}(G)$. Then the G-signature Sign: $\tilde{L}_{2 n}^{s}(G) \rightarrow \mathbf{Z} \hat{G}$ is injective with image $\left\{4 \sum_{r=1}^{(q-1) / 2} a_{r}\left(\chi^{r}+(-1)^{n} \chi^{-r}\right) \mid a_{r} \in\right.$ $\mathbf{Z}\}$. 
As for $R_{\hat{G}}$, there is an augmentation $\varepsilon^{\prime}: R_{\hat{G}} \rightarrow \mathbf{Z}_{q}$ induced from the augmentation $\varepsilon: \mathbf{Z} \hat{G} \rightarrow \mathbf{Z}$ taking each $\chi^{i} \in \hat{G}$ to 1 . Let $I_{\hat{G}}$ be the kernel of $\varepsilon^{\prime}$; the kernel of $\varepsilon$ maps isomorphically to $I_{\hat{G}}$ under the natural projection $\mathbf{Z} \hat{G} \rightarrow R_{\hat{G}}$. Also, $I_{\hat{G}}$ is a principal ideal with generator $(\chi-1)$. This generator is a unit of $R_{\hat{G}}[1 / q]$, so $I_{\hat{G}}$ is an invertible $R_{\hat{G}}$-submodule of $R_{\hat{G}}[1 / q]$. For any $n \in \mathbf{Z}, I_{\hat{G}}^{n} / I_{\hat{G}}^{n+1} \approx \mathbf{Z}_{q}$. Finally, the involution on $\mathbf{Z} \hat{G}$ taking $\chi^{i}$ to $\chi^{-i}$ passes to an involution on $R_{\hat{G}}$. We say $y \in R_{\hat{G}}$ is real if $\bar{y}=y$ and imaginary if $\bar{y}=-y$. Re stands for "real elements of" and similarly for Im.

Now we treat the case $m=2 k \geqslant 4$. The following result is essentially [25, 14E.6].

Proposition 4.8. Let

$$
\sigma^{\prime}:\left[\left(D^{4 k}, S^{4 k-1}\right) \times L^{2 d-1}, F / \mathrm{PL}\right] \rightarrow R_{\hat{G}}[1 / q] / R_{\hat{G}}
$$

be induced by $\sigma$. Then $\sigma^{\prime}$ takes the summand $\left[\Sigma^{4 k} L, F / \mathrm{PL}\right]$ isomorphically to $\operatorname{Re} I_{\hat{G}}^{-d+2} / \operatorname{Re} R_{\hat{G}}$ if $d$ is even and to $\operatorname{Im} I_{\hat{G}}^{-d+2} / \operatorname{Im} R_{\hat{G}}$ if $d$ is odd. Each normal cobordism class in $\left[\Sigma^{4 k} L, F / \mathrm{PL}\right]$ contains a simple homotopy equivalence with $\sigma$ divisible by 4 and $s_{0}=0$.

Proof. We use induction on $d$ to prove injectivity. For $d \leqslant 2,\left[\Sigma^{4 k} L, F / \mathrm{PL}\right]=0$ by 4.1. If $d \geqslant 3$, suppose inductively that we have simple homotopy triangulations $h_{i}: N_{i} \rightarrow D^{4 k} \times L^{2 d-3}, 0 \leqslant i \leqslant q^{[(d-2) / 2]}$ such that (a) $\sigma\left(h_{i}\right) \in I_{\hat{G}}^{-(d-1)+2}$ and (b) if $i \neq j, \sigma\left(h_{i}\right) \neq \sigma\left(h_{j}\right) \bmod R_{\hat{G}}$. From these, we shall construct triangulations of $D^{4 k} \times L^{2 d-1}$ satisfying (a) and (b) with $d$ in place of $d-1$.

Let $* S^{1}$ be suspension by the action of $G$ on $S^{1}$ via $\chi^{p}$. Then $\sigma\left(h_{i} * S^{1}\right)=$ $\sigma\left(h_{i}\right) \sigma\left(S^{1}\right)=\sigma\left(h_{i}\right)\left(\chi^{p}+1\right) /\left(\chi^{p}-1\right) \in 4 I_{\hat{G}}^{-d+2}$. If $\sigma\left(h_{i} * S^{1}\right) \equiv \sigma\left(h_{j} * S^{1}\right)$ $\bmod R_{\hat{G}}$ then $\sigma\left(h_{i}\right) \equiv \sigma\left(h_{j}\right) \bmod R_{\hat{G}}$, so (b) holds for the $h_{i} * S^{1}$.

When $d$ is even, the orders of $\left[\Sigma^{4 k} L^{2 d-3}, F / \mathrm{PL}\right]$ and $\left[\Sigma^{4 k} L^{2 d-1}, F / \mathrm{PL}\right]$ are equal, so the $h_{i} * S^{1}$ satisfy (a) and (b) with $d$ in place of $d-1$ and are sufficient in number. When $d$ is odd, act upon each $h_{i}$ by $\varphi_{j} \in \tilde{L}_{4 k+2 d-2}^{s}(G)$ with $\operatorname{Sign}\left(G, \varphi_{j}\right)=$ $4 j\left(\chi+\chi^{-1}\right), 0 \leqslant j<q$. This is possible since $d \geqslant 3$ implies $\pi_{1} L^{2 d-3} \approx G$, so 4.7 applies. We get new triangulations $\varphi_{j} h_{i}$ with $\sigma\left(\varphi_{j} h_{i}\right)=\sigma\left(h_{i}\right)+4 j\left(\chi+\chi^{-1}\right)$. Now form $\varphi_{j} h_{i} * S^{1}$. Then (a) holds.

We claim that if $i \neq s$ or $j \neq t$, then $\sigma\left(\varphi_{j} h_{i} * S^{1}\right)-\sigma\left(\varphi_{t} h_{s} * S^{1}\right) \notin R_{\hat{G}}$. For if $i \neq s, \varphi_{j} h_{i}$ and $\varphi_{t} h_{s}$ have distinct $\sigma \bmod R_{\hat{G}}$, so their suspensions do, too. If

$$
\sigma\left(\varphi_{j} h_{i} * S^{1}\right)-\sigma\left(\varphi_{t} h_{i} * S^{1}\right)=4(j-t)\left(\chi+\chi^{-1}\right)\left(\chi^{p}+1\right) /\left(\chi^{p}-1\right) \in R_{\hat{G}}
$$

then $4(j-t) \in I_{\hat{G}}=\operatorname{ker} \varepsilon^{\prime}$, so we must have $j=t$.

Hence $\sigma^{\prime}$ is injective. Since $\operatorname{Re} I_{\hat{G}}^{-d+2} / \operatorname{Re} R_{\hat{G}}$ has order $q^{[(d-1) / 2]}$ if $d$ is even (similarly if $d$ is odd), the image of $\sigma^{\prime}$ is as claimed. Finally, the triangulations all have $s_{0}=0$ since they were obtained from the identity map of $D^{4 k} \times L^{3}$ by normal cobordisms and suspensions and these do not alter $s_{0}$.

COROLlaRY 4.9. The homomorphism

$$
\left(\sigma, s_{0}\right): S_{\mathrm{PL}}^{s}\left(\left(D^{4 k}, S^{4 k-1}\right) \times L^{2 d-1}\right) \rightarrow R_{\hat{G}}[1 / q] \oplus \mathbf{Z}
$$

is injective and $\sigma$ takes the kernel of $s_{0}$ isomorphically to $4 \operatorname{Re} I_{\hat{G}}^{-d+2}$ (respectively $4 \operatorname{Im} I_{\hat{G}}^{-d+2}$ ) if $d$ is even (respectively odd). 
Proof. The second assertion implies the first. To prove it, note that the $\sigma$ invariants of two normally cobordant simple homotopy triangulations differ by an element of $4 R_{\hat{G}}$ and $4 I_{\hat{G}}^{-d+2} / 4 R_{\hat{G}} \approx I_{\hat{G}}^{-d+2} / R_{\hat{G}}$, so surjectivity follows from 4.8 . Injectivity follows from the surgery sequence (4.6) and 4.7.

Thus $S_{\mathrm{PL}}^{s}$ is free abelian of rank $(q+1) / 2$ (by (4.6)), so its image under $\left(\sigma, s_{0}\right)$ is of finite index in $\operatorname{Re} R_{\hat{G}} \oplus \mathbf{Z}$ ( $d$ even) or $\operatorname{Im} R_{\hat{G}} \oplus \mathbf{Z}$ ( $d$ odd). To compute it, we look at $\sigma^{\prime}$ on $\pi_{4 k} F /$ PL.

LEMMA 4.10. If $k \geqslant 2$ there is a generator of $\pi_{4 k} F / \mathrm{PL}$ whose $\sigma^{\prime}$ invariant is $8 \sigma(L)$ $\bmod R_{\hat{G}}$.

Proof. Let $M^{4 k}$ be a Milnor manifold of signature 8 and $g:(M, \partial M) \rightarrow$ $\left(D^{4 k}, S^{4 k-1}\right)$ a degree 1 normal map with $\partial g$ a PL homeomorphism. Then $g \times \mathrm{Id}_{L}$ is a normal map and $s_{0}\left(g \times \mathrm{Id}_{L}\right)=1$. Also, $\sigma\left(g \times \mathrm{Id}_{L}\right)=\sigma(M \times L)=$ Sign $M \cdot \sigma(L)=8 \sigma(L)$. This completes the proof.

Do surgery on $g \times \operatorname{Id}_{L}$ to get a simple homotopy equivalence $h_{0}$. Then $s_{0}\left(h_{0}\right)=1$ and $\sigma\left(h_{0}\right)=8 \sigma(L)+\psi$, where $\psi \in R_{\hat{G}}$ is the $G$-signature of the trace of the surgery. $h_{0}$ generates an infinite cyclic subgroup $H$ of $S_{\mathrm{PL}}^{s}$ mapped isomorphically to $\mathbf{Z}$ by $s_{0}$. So $S_{\mathrm{PL}}^{s}\left(\left(D^{4 k}, S^{4 k-1}\right) \times L\right) \approx H \oplus \operatorname{ker} s_{0}$ and the image of $\left(\sigma, s_{0}\right)$ lies in $I_{\hat{G}}^{-d} \oplus \mathbf{Z}$ and is the sum of $4 \operatorname{Re} I_{\hat{G}}^{-d+2}$ and the subgroup generated by $(8 \sigma(L)+\psi, 1)$.

THEOREM 4.11. If $k \geqslant 2, \sigma$ and $s_{0}$ determine each element of $S_{\mathrm{PL}}^{s}\left(\left(D^{4 k}, S^{4 k-1}\right) \times\right.$ $\left.L^{2 d-1}\right)$ uniquely. $\sigma$ takes values in $I_{\hat{G}}^{-d}$ and is real or imaginary as $d$ is even or odd. The only relations between $\sigma$ and $s_{0}$ are the $(\bmod q)$ relation

$$
\sigma \equiv 8 \sigma(L) s_{0} \quad \bmod I_{\hat{G}}^{-d+2}
$$

and the 2-primary relation

$$
\sigma \equiv \psi s_{0} \quad \bmod 4 I_{\hat{G}}^{-d} .
$$

Proof. All but the last assertion have been established. By a Chinese Remainder Theorem argument, the two relations are equivalent to $\sigma \equiv(8 \sigma(L)+\psi) s_{0}$ $\bmod 4 I_{\hat{G}}^{-d+2}$ and so our assertion follows from the sentence before the theorem.

THEOREM 4.12. Let $k \geqslant 2$. If $h_{i}: N_{i} \rightarrow D^{4 k} \times L, i=1,2$, are two homotopy triangulations for which $\sigma, \tau$ (Whitehead torsion), and $s_{0}$ agree, there is a PL homeomorphism $N_{1} \rightarrow N_{2}$.

Proof. Since $s_{0}$ and $\sigma^{\prime}$ of the $h_{i}$ agree, $h_{1}$ is normally cobordant to $h_{2}$ by some $W \rightarrow D^{4 k} \times L \times I$. That is, $h_{2}$ is obtained from $h_{1}$ by the action of some $\varphi \in$ $\tilde{L}_{4 k+2 d}^{h}(G)$. Since $\operatorname{Sign}(G, \varphi)=\sigma\left(h_{2}\right)-\sigma\left(h_{1}\right)=0$ and $D(\varphi)=\tau^{\prime}\left(h_{2}\right)-\tau^{\prime}\left(h_{1}\right)=$ $0, \varphi$ is zero by the Rothenberg sequence of 2.5. Thus $W$ is an $h$-cobordism and since $\tau\left(h_{1}\right)=\tau\left(h_{2}\right)$, it is an $s$-cobordism. This completes the proof.

Whitehead torsion is related to the Atiyah-Singer invariant, but only by a 2-primary relation since one may change the torsion of a triangulation by any even element of Wh $G$ using an $h$-cobordism and this does not change $\sigma$. The connection occurs in the Wall group $L_{2 n}^{h}(G)$ as follows. Let $W G$ be the subgroup of real units of $\mathrm{Z} G$ whose augmentation is 1 . Then $W G \approx \mathrm{Wh} G$ by [25, 14E.2], so we identify the 
two. For each integer $r$, the character $\chi^{r}$ extends to a ring map $\mathbf{Z} G \rightarrow \mathbf{Z}[\lambda]$ taking $W G$ to $\mathbf{Z}\left[\lambda+\lambda^{-1}\right]^{*}$. Let sgn: $\mathbf{Z}\left[\lambda+\lambda^{-1}\right]^{*} \rightarrow\{ \pm 1\}$ be the signum function. Then $\operatorname{sgn} \circ \chi^{r}: W G \rightarrow\{ \pm 1\}$ factors through Wh $G / 2 \mathrm{Wh} G$, so we define

$$
S: \mathrm{Wh} G / 2 \mathrm{Wh} G \rightarrow 2 \operatorname{Re} \mathbf{Z} \hat{G}
$$

by $S(w)=\sum_{r=1}^{q-1}\left[\operatorname{sgn}\left(\chi^{r}(w)\right)-1\right] \chi^{r}$. According to $[25,13 \mathrm{~A} .5]$, the only relation between $\operatorname{Sign}$ and $D$ on $L_{2 n}^{h}(G)$ is $\operatorname{Sign}(G, \varphi) \equiv S(D(\varphi))$ mod 4. This translates into $\sigma(h) \equiv S\left(\tau^{\prime}(h)\right) \bmod 4 R_{\hat{G}}$ if $h$ lies in the image of

$$
\tilde{L}_{4 k+2 d}^{h}(G) \rightarrow S_{\mathrm{PL}}^{h}\left(\left(D^{4 k}, S^{4 k-1}\right) \times L\right) .
$$

Proposition 4.13. If $h$ is a homotopy triangulation,

$$
\sigma(h)-S\left(\tau^{\prime}(h)\right)-\psi s_{0}(h) \in 4 I_{\hat{G}}^{-d} .
$$

Proof. $h$ is normally cobordant to a simple triangulation $k$ since $L_{\text {odd }}^{s}(G)=0$. Write $h=\varphi k$ as in 4.8, $\varphi \in \tilde{L}_{4 k+2 d}^{h}(G)$. Then $\sigma(h)=\operatorname{Sign}(G, \varphi)+\sigma(k) \equiv S(D(\varphi))+\psi s_{0}(k)=S\left(\tau^{\prime}(h)\right)+\psi s_{0}(h) \bmod 4 I_{\hat{G}}^{-d}$.

THEOREM 4.14. If $k \geqslant 2$, each $H \in S_{\mathrm{PL}}^{h}\left(\left(D^{4 k}, S^{4 k-1}\right) \times L^{2 d-1}\right)$ is uniquely determined by $\sigma(H), \tau^{\prime}(H)$, and $s_{o}(H)$. The relations

$$
\sigma \equiv 8 \sigma(L) s_{0} \quad \bmod I_{\hat{G}}^{-d+2}
$$

and

$$
\sigma \equiv S\left(\tau^{\prime}\right)+\psi s_{0} \quad \bmod 4 I_{\hat{G}}^{-d}
$$

and the only relations between $\sigma, \tau^{\prime}$, and $s_{0}$ valid on all of $S_{\mathrm{PL}}^{h}$.

Proof. We need only prove that if $a \in I_{\hat{G}}^{-d}, n \in \mathbf{Z}$, and $w \in$ Wh $G$ satisfy $a \equiv(8 \sigma(L)+\psi) n+S(w) \bmod 4 I_{\hat{G}}^{-d+2}$, then $a, n$, and $w$ are realized by a triangulation. Let $h$ be a triangulation such that $\tau(h)=w(\mathrm{cf}$. 2.5). Then

$$
\begin{aligned}
\sigma(h) & \equiv(8 \sigma(L)+\psi) s_{0}(h)+S(w) \\
& \equiv(8 \sigma(L)+\psi) s_{0}(h)+a-(8 \sigma(L)+\psi) n \bmod 4 I_{\hat{G}}^{-d+2},
\end{aligned}
$$

so $a-\sigma(h) \equiv(8 \sigma(L)+\psi)\left(n-s_{0}(h)\right) \bmod 4 I_{\hat{G}}^{-d+2}$. By 4.11 , there is a simple homotopy triangulation $k$ with $\sigma(k)=a-\sigma(h)$ and $s_{0}(k)=n-s_{0}(h)$. Hence $\left(\sigma, s_{0}, \tau^{\prime}\right)(k+h)=(a, n, w)$.

REMARKS. 1. Since $h_{0}$ is determined only up to the action of $L_{4 k+2 d}^{s}(G), \psi$ is well defined only modulo $4 R_{\hat{G}}$. In general, I do not knows its mod 4 coset. However, when $u=d \chi, \psi \in 4 R_{\hat{G}}$ so it disappears from our relations. This result depends on a good deal of work on triangulations of $D^{4 k} \times($ complex projective $(d-1)$-space) and will not be discuissed here.

2. We shall not discuss the case $m=2 k+1$. One obtains results like those above, except that the lowest dimensional case of 4.8 must be assumed in order to begin the induction. However, if $u=d \chi$, this assumption is met. 
Our last result concerns the suspension

$$
* S^{1}: S_{\mathrm{PL}}^{c}\left(\left(D^{4 k}, S^{4 k-1}\right) \times L^{2 d-1}\right) \rightarrow S_{\mathrm{PL}}^{c}\left(\left(D^{4 k}, S^{4 k-1}\right) \times L^{2 d+1}\right)
$$

by the action of $G$ on $S^{1}$ via $\chi^{p}$, where $(p, q)=1$.

THEOREM 4.15. If $k \geqslant 2$ and $d \geqslant 2, * S^{1}$ is an isomorphism.

Proof. Recall that under suspension, $\sigma$ is multiplied by

$$
\sigma\left(S^{1}\right)=\left(\chi^{p}+1\right) /\left(\chi^{p}-1\right)
$$

and $s_{0}$ and $\tau$ are unchanged. Now $\sigma\left(S^{1}\right)$ is a unit of $R_{\hat{G}}[1 / q]$ by $[25$, p. 205] and $\left(\sigma, s_{0}, \tau^{\prime}\right)$ is injective, so $* S^{1}$ is injective. For surjectivity, let $h \in$ $S_{\mathrm{PL}}^{c}\left(\left(D^{4 k}, S^{4 k-1}\right) \times L^{2 d+1}\right)$ and $(a, n, w)=\left(\sigma(h), s_{0}(h), \tau^{\prime}(h)\right)$. Then

$$
a \equiv 8 \sigma\left(L^{2 d-1}\right) \sigma\left(S^{1}\right) n \bmod I_{\hat{G}}^{-d+1}
$$

and

$$
a \equiv S(w)+\psi(d+1) n \bmod 4 I_{\hat{G}}^{-d-1}
$$

where $\psi(d+1)$ is the $\psi$ corresponding to $L^{2 d+1}$. Hence

$$
\begin{aligned}
& \sigma\left(S^{1}\right)^{-1} a \equiv 8 \sigma\left(L^{2 d-1}\right) n \bmod I_{\hat{G}}^{-d+2}, \\
& \sigma\left(S^{1}\right)^{-1} a \equiv \sigma\left(S^{1}\right)^{-1} S(w)+\sigma\left(S^{1}\right)^{-1} \psi(d+1) n \bmod 4 I_{\hat{G}}^{-d}
\end{aligned}
$$

and all elements are suitably real or imaginary since $\sigma\left(S^{1}\right)$ is imaginary. Now $\sigma\left(S^{1}\right)^{-1}+1=2 \chi^{p} /\left(\chi^{p}+1\right)$ and $S(w) \in 2 R_{\hat{G}}$, so $\sigma\left(S^{1}\right)^{-1} S(w) \equiv-S(w) \equiv S(w)$ $\bmod 4 R_{\hat{G}}$. Thus $\sigma\left(S^{1}\right)^{-1} a \equiv S(w)+\sigma\left(S^{1}\right)^{-1} \psi(d+1) n \bmod 4 I_{\hat{G}}^{-d}$. So as long as $\sigma\left(S^{1}\right)^{-1} \psi(d+1) \equiv \psi(d) \bmod 4 I_{\hat{G}}^{-d},\left(\sigma\left(S^{1}\right)^{-1} a, n, w\right)$ will occur as the invariants of an element of $S_{\mathrm{PL}}^{c}\left(\left(D^{4 k}, S^{4 k-1}\right) \times L^{2 d-1}\right)$ and we will be done.

To see this, let $h_{0}$ be as after 4.10 so that $\sigma\left(h_{0}\right)=\psi(d)+8 \sigma\left(L^{2 d-1}\right)$. Then $\sigma\left(h_{0} * S^{1}\right)=\sigma\left(S^{1}\right) \psi(d)+8 \sigma\left(L^{2 d+1}\right)$ and $s_{0}\left(h_{0} * S^{1}\right)=1$. Hence $\sigma\left(S^{1}\right) \psi(d) \equiv$ $\psi(d+1) \bmod 4 I_{\hat{G}}^{-d+1}$. This completes the proof.

REMARK. The theorem is valid if $m=2 k+1 \geqslant 3$ and $u$ is such that the analog of 4.8 holds, e.g. if $u=d \chi$.

5. Applications. Let $\alpha=(2 m-1) \oplus u, m \geqslant 3$. We shall use our results on $S_{\mathrm{PL}}^{h}\left(\left(D^{4 k}, S^{4 k-1}\right) \times L\right)$ and the homomorphism $f$ to study $\alpha$-spheres. Our groups are linked by the braid:

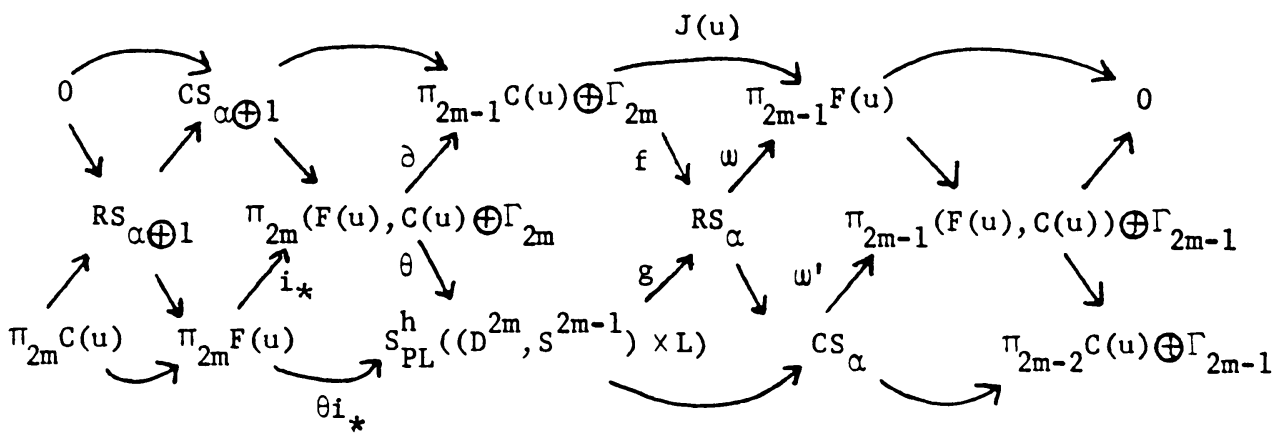


The two zero groups are from 4.2. $\Gamma_{n}$ is the group of $h$-cobordism classes of homotopy $n$-spheres. $F(u)$ is the space of self $G$-maps of $S(u)$. The map $g$ was treated in $\S 4$ and $f$ on $\pi_{n} C(u)$ in $\S 3$. If $\Sigma^{n+1}$ is a homotopy $(n+1)$-sphere constructed by glueing two disks together with a diffeomorphism $j$ of $S^{n}$ and $h$ : $S^{n} \rightarrow C(u)$, then $f(h, \Sigma)$ is the standard $\alpha$-sphere with trivialization $\hat{h} \circ(j \times$ Id $), \hat{h}$ as in the definition of $f$ after 3.2. $\theta(\Sigma): D^{n+1} \times L \rightarrow D^{n+1} \times L$ is just $\tilde{j} \times \operatorname{Id}_{L}$ where $\tilde{j}: D^{n+1} \rightarrow D^{n+1}$ extends $j . \theta$ is described on $\pi_{n+1}(F(u), C(u))$ in the proof of 5.2. $\omega$ and $\omega^{\prime}$ are the knot invariants of $\left[16 ; 18\right.$, pp. 310-311]. The map $C S_{\alpha} \rightarrow \pi_{2 m-2} C(u)$ $\oplus \Gamma_{2 m-1}$ classifies the fixed set and its normal bundle in the $\alpha$-sphere. The sequences involving $f$ and $\omega$ are from [16,5]; Schultz [18] derived that involving $\omega^{\prime}$ from them. Recall that $C(u) \approx \prod_{k \in A} U\left(d_{k}\right)$. Thus $\pi_{\text {even }} C(u)$ are finite and $\pi_{2 m-1} C(u)$ has rank equal to the number of $d_{k} \geqslant m$.

Proposition 5.1. If $n \neq 2 d-1$, then $\pi_{n}(F(u)$,Id) is finite. Moreover, if $n<2 d-2$, there is a homomorphism $i_{*}: \pi_{n} F(u) \rightarrow \pi_{n}^{s}$ to the stable $n$-stem whose kernel and cokernel are q-groups.

Proof. Let $F(u, \tilde{p}) \subset F(u)$ be the space of $G$ maps preserving a basepoint $\tilde{p}$. We shall compare $F(u, \tilde{p})$ to the loop space $\Omega^{2 d-1} S^{2 d-1}$ and $F(u)$ to $F(u, \tilde{p})$ to prove the proposition.

Let $\pi: S^{2 d-1} \rightarrow L$ be the covering projection and $p=\pi(\tilde{p})$. The identity component of $F(u, \tilde{p})$ is homeomorphic to the identity component of the $\operatorname{space} \operatorname{Map}(L, p)$ of pointed self-maps of $L$, so $\pi_{*} F(u, \tilde{p}) \approx \pi_{*} \operatorname{Map}(L, p)$. Forgetting the $G$-action induces an inclusion $i$ : $F(u, \tilde{p}) \hookrightarrow \Omega^{2 d-1} S^{2 d-1}$ which we analyze using the commutative diagram:

$$
\begin{array}{ccc}
\operatorname{Map}(L, p) \approx F(u, \tilde{p}) & \stackrel{i}{\rightarrow} & \Omega^{2 d-1} S^{2 d-1} \\
\pi^{*} \searrow & & \swarrow \Omega^{2 d-1} \pi \\
& \Omega^{2 d-1} L &
\end{array}
$$

$\Omega^{2 d-1} \pi$ induces an isomorphism on $\pi_{*}$ since $\pi$ does for $*>1$. By elementary obstruction theory, the kernel and cokernel of the map induced by $\pi^{*}$ on $\pi_{n}$ are $q$-groups. Thus $i_{*}$ is an isomorphism modulo $q$-torsion. Recall that $\pi_{n} \Omega^{2 d-1} S^{2 d-1}$ is always finite and isomorphic to $\pi_{n}^{s}$ if $n<2 d-2$ [23].

Now the homotopy sequence of the evaluation (at $\tilde{p})$ fibration $F(u, \tilde{p}) \rightarrow F(u) \rightarrow$ $S^{2 d-1}$ shows that $\pi_{n} F(u, \tilde{p}) \rightarrow \pi_{n} F(u)$ is an isomorphism if $n<2 d-2$ and an isomorphism modulo torsion if $n \neq 2 d-1$. Putting these results together completes the proof.

LEMMA 5.2. The homomorphism $\theta$ factors through $S_{\mathrm{PL}}^{s}\left(\left(D^{n+1}, S^{n}\right) \times L\right)$. Hence the torsion subgroup of $\pi_{4 k}(F(u), C(u)) \oplus \Gamma_{4 k}$ lies in the kernel of $\theta$. In particular, $\theta i_{*}$ is zero on $\pi_{4 k} F(u)$.

Proof. If $\gamma:\left(D^{n+1}, S^{n}, p t\right) \rightarrow(F(u), C(u)$, Id $)$, then $\theta(\gamma): D^{n+1} \times L \rightarrow D^{n+1} \times L$ is induced by $(x, y) \mapsto(x, \gamma(x)(y))$. Since

$$
p t \times L \subset D^{n+1} \times L \stackrel{\theta(\gamma)}{\rightarrow} D^{n+1} \times L \stackrel{\pi_{2}}{\rightarrow} L
$$


is the identity and the first and last maps are simple, $\theta(\gamma)$ is a simple homotopy equivalence. Next, if $\Sigma^{n+1}=D^{n+1} \cup_{j} D^{n+1}$ is a homotopy sphere, $\tau(\theta(\Sigma))=0$ since the extension $\tilde{j}: D^{n+1} \rightarrow D^{n+1}$ is necessarily simple. So $\theta$ factors through the simple homotopy triangulations.

The remaining assertions are true because $S_{\mathrm{PL}}^{s}\left(\left(D^{4 k}, S^{4 k-1}\right) \times L\right)$ is free abelian (4.9) and $\pi_{4 k} F(u)$ is finite.

COROllary 5.3. Let $\alpha=(2 m-1) \oplus u, m \geqslant 2$. Then $S_{\alpha \oplus 1} \approx C S_{\alpha \oplus 1}$ is finite. If $m=2 k$, then $C S_{\alpha \oplus 1} \approx \operatorname{Tor}\left(\pi_{4 k}(F(u), C(u)) \oplus \Gamma_{4 k}\right)$ and $R S_{\alpha \oplus 1} \approx \pi_{4 k} F(u)$.

Proof. Modulo torsion, $\partial$ and $f$ are both injective, the latter by 3.4. Thus, the same holds for $\theta$ and so $C S_{\alpha \oplus 1}=\operatorname{ker} \theta$ is finite. That $S_{\alpha \oplus 1}$ is isomorphic to $C S_{\alpha \oplus 1}$ is contained in the proof of 2.5 .

If $m=2 k$, then the injectivity of $\theta$ modulo torsion and 5.2 show that the kernel of $\theta$ is the torsion subgroup of $\pi_{4 k}(F(u) C(u)) \oplus \Gamma_{4 k}$.

REMARK. The finiteness result is due to Ewing, who proved the linear independence of the $\Phi_{m}\left(\lambda^{k}\right)$ in [8] in order to obtain it.

Now we turn to $\alpha$-spheres, where $\alpha=(4 k-1) \oplus u$ and $d \neq 2 k$. Then $g$ is a rational isomorphism, so there is a unique extension of $s_{0}$ on $S_{\mathrm{PL}}^{h}$ to $s_{0}: R S_{\alpha} \rightarrow \mathbf{Q}$.

THEOREM 5.4. If $\alpha=(4 k-1) \oplus u, k \geqslant 2$, and $k \neq 2 d$, then $R S_{\alpha} \otimes \mathbf{Q}$ and $C S_{\alpha} \otimes \mathbf{Q}$ are completely described by $\sigma$ and $s_{0}$, and $S_{\alpha} \otimes \mathbf{Q}$ by these two invariants and $\tau_{\alpha}$.

Proof. Because $g \otimes \mathbf{Q}$ is an isomorphism, $\left(\sigma, s_{0}\right)$ takes $R S_{\alpha} \otimes \mathbf{Q}$ isomorphically to $\operatorname{Re} \mathbf{Q} R_{\hat{G}} \oplus \mathbf{Q}$ or $\operatorname{Im} \mathbf{Q} R_{\hat{G}} \oplus \mathbf{Q}$ as $d$ is even or odd. $C S_{\alpha} \otimes \mathbf{Q}$ is the quotient of $R S_{\alpha} \otimes \mathbf{Q}$ by the image of $f \otimes \mathbf{Q}$. We already have a formula for $\sigma \circ f$ on $\pi_{4 k-1} C(u)$ and shall shortly have one for $s_{0} \circ f$, so we can exhibit a basis for $\left(\sigma, s_{0}\right)$ (image $\left.f \otimes \mathbf{Q}\right)$ and thus describe $C S_{\alpha} \otimes \mathbf{Q}$. Finally, we have the sequence

$$
0 \rightarrow \mathrm{Wh} G \rightarrow S_{\alpha} \rightarrow C S_{\alpha} \rightarrow 0
$$

from the proof of 2.5 , and $\tau_{\alpha} \otimes \mathbf{Q}$ splits Wh $G \otimes \mathbf{Q} \rightarrow S_{\alpha} \otimes \mathbf{Q}$.

REMARKS. When $m=2 k+1$, there are slightly weaker counterparts of 5.2-5.4. In this case, the splitting invariant $s_{0}$ is $\mathbf{Z}_{2}$-valued so disappears rationally.

Because the smooth analogues of our PL groups differ from the latter by torsion, Theorem 5.4 describes their rational structure, too. Browder and Petrie computed $R S_{\alpha} \otimes \mathbf{Q}$ using $\left(\sigma, s_{0}\right)$ in [5, Theorem 4] and lacked only Ewing's result in their description of $C S_{\alpha} \otimes \mathbf{Q}$.

Explicit calculation of $R S_{\alpha}$ is not possible in general. We have determined the behavior of our invariants on the subgroup (image $f+$ image $g$ ) of $R S_{\alpha}$. $R S_{\alpha}$ /(image $f+$ image $g$ ) is isomorphic to the cokernel of the equivariant $J$-homomorphism $J(u)$ in our braid diagram, so it is presently intractable. Spheres in $R S_{\alpha}$ - (image $f+$ image $g$ ) are very difficult to construct, as $f$ accounts for changes of trivialization, and $g$ for other constructive techniques including surgery on the free part and surgery on the fixed set preserving the trivialization. Yet such spheres exist; see 5.12 below for some examples which are even smooth.

On the other hand, we can estimate the image of $J(u)$. For the relations between our invariants on $S_{\mathrm{PL}}^{h}$ may be viewed as integrality conditions on characteristic 
numbers which give rise to invariants on $\pi_{4 k-1} F(u)$. To begin, we introduce a normalized version of $\sigma$. Let

$$
\mu=\sigma\left(L^{2 d-1}\right)^{-1} \cdot \sigma: R S_{\alpha} \rightarrow R_{\hat{G}}[1 / q]
$$

$(2 d=\operatorname{dim} u)$. Then $\mu$ is real-valued.

INTEGRALITY THEOREM 5.5. If $k \geqslant 2$, then the invariants $\mu$ and $s_{0}: R S_{\alpha} \rightarrow \mathbf{Q}$ satisfy the following conditions on the subgroup $g\left(S_{\mathrm{PL}}^{h}\left(\left(D^{4 k}, S^{4 k-1}\right) \times L\right)\right)$ :

(a) $\mu$ takes values in the real elements of $R_{\hat{G}}$.

(b) $s_{0}$ takes values in the integers.

(c) $\mu-8 s_{0}$ takes values in the real elements of $I_{\hat{G}}^{2}$.

Thus we have homomorphisms:

(a) $\bar{\mu}: \pi_{4 k-1} F(u) \rightarrow R_{\hat{G}}[1 / q] / R_{\hat{G}}$.

(b) $\bar{s}_{0}: \pi_{4 k-1} F(u) \rightarrow \mathbf{Q} / \mathbf{Z}$.

(c) $\bar{\mu}-8 \bar{s}_{0}: \pi_{4 k-1} F(u) \rightarrow \mathbf{Q} R_{\hat{G}} / I_{\hat{G}}^{2}$.

Proof. The three conditions are simply reformulations of 4.14. The homomorphisms are defined using the exact sequence for $g$, in which $\omega: R S_{\alpha} \rightarrow \pi_{4 k-1} F(u)$ is surjective.

REMARKS. Calling (a) an integrality condition is a natural thing to do if one observes that, under the homomorphism $\mathbf{Q} R_{\hat{G}} \rightarrow \mathbf{Q}(\lambda)$ sending $\chi$ to $\lambda, R_{\hat{G}}$ is taken to the ring $\mathbf{Z}[\lambda]$ of algebraic integers.

The second relation of $4.14, \sigma \equiv S\left(\tau^{\prime}\right)+\psi s_{0} \bmod 4 I_{\hat{G}}^{-d}$, gives a homomorphism

$$
\bar{\sigma}-\bar{S}\left(\tau_{\alpha}\right): \pi_{4 k-1}(F(u), C(u)) \rightarrow R_{\hat{G}}[1 / q] / 4 R_{\hat{G}}[1 / q]
$$

if $\psi \in 4 R_{\hat{G}}$ since $\sigma \circ f \in 4 R_{\hat{G}}[1 / q]$ by 3.6 and $\tau_{\alpha} \circ f=0$. I have not yet had success computing it.

To study $\bar{s}_{0}$, we now define $s_{0}$ geometrically. In [26], an invariant $l$ of a manifold $M^{4 k-1}$ with framing $t$ is defined by $l(M, t)=L(W)[W]-\operatorname{sign} W$ where $\partial W=M$ and $L(W)$ is the Hirzebruch class of $W$, made into a relative class using $t$. Given $(\Sigma, t)$ representing an element of $R S_{\alpha}$, some multiple $N(\Sigma, t)=\left(S^{4 k-1+2 d}, t^{\prime}\right)$ and we set $l(\Sigma, t)=l\left(S^{4 k-1+2 d}, t^{\prime}\right) / N$. Then $s_{0}=-l$.

LEMMA 5.6. Let $S G(2 d)$ be the space of degree 1 self-maps of $S^{2 d-1}$ and $i$ : $F(u) \rightarrow S G(2 d)$ the inclusion. Then there is a factorization:

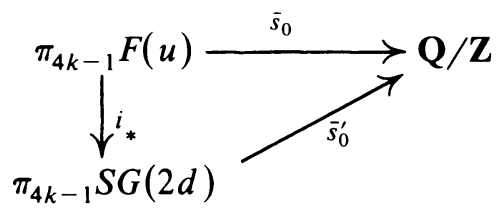

Moreover, if $d>2 k, \bar{s}_{0}^{\prime}$ maps the odd primary part of the image of $\mathrm{J}: \pi_{4 k-1} S O(2 d)$ $\rightarrow \pi_{4 k-1} S G(2 d)$ monomorphically into $\mathbf{Q} / \mathbf{Z}$.

Proof. The factorization comes about because there is a map from the exact sequence of $g$ to its nonequivariant analogue. Now $l$ induces a homomorphism $\pi_{4 k-1+2 d} S^{2 d} \rightarrow \mathbf{Q} / \mathbf{Z}$. By [27, p. 215], this coincides at odd primes with Adams' $e$-invariant [1], so $\bar{s}_{0}^{\prime}$ detects the odd primary part of the image of $J$ when $d>2 k$. 
For later use we shall need an explicit formula for $s_{0} \circ f$. To obtain it we use the diagram

$$
\begin{array}{ccc}
\pi_{4 k}(S G(2 d), S O(2 d)) & \stackrel{\partial}{\rightarrow} & \pi_{4 k-1} S O(2 d) \\
\downarrow \theta & & \downarrow f \\
S_{\mathrm{PL}}\left(\left(D^{4 k}, S^{4 k-1}\right) \times S^{2 d-1}\right) & \stackrel{g}{\rightarrow} & R S_{\alpha}(1) \stackrel{s_{0}^{\prime}}{\rightarrow} \mathbf{Q}
\end{array}
$$

which is just the nonequivariant version of a square in our braid diagram. Let $\gamma \in \pi_{4 k}(S G(2 d), S O(2 d)), d>2 k$. Then $s_{0}^{\prime}(f(\partial \gamma))=s_{0}^{\prime}(\theta(\gamma))=\left(\operatorname{Sign} M^{\prime}\right) / 8$, where $M^{\prime}$ is obtained by making $\theta(\gamma)$ transverse to $D^{4 k} \times p t$. Now $\gamma$ gives rise to a fiber homotopy trivialization

$$
\Gamma: E=D^{4 k} \times \mathbf{R}^{2 d} \cup_{\partial \gamma} D^{4 k} \times \mathbf{R}^{2 d} \stackrel{\gamma \cup \mathrm{Id}}{\rightarrow} S^{4 k} \times \mathbf{R}^{2 d} \stackrel{\pi_{2}}{\rightarrow} \mathbf{R}^{2 d}
$$

of the $\mathbf{R}^{2 d}$-bundle $E \rightarrow S^{4 k}$ corresponding to $\gamma$. Clearly Sign $M^{\prime}=\operatorname{Sign} M$, where $M=\Gamma^{-1}(0)$ is obtained by making $\Gamma$ transverse to $0 \in \mathbf{R}^{2 d}$. Now $h: M \hookrightarrow E \rightarrow S^{4 k}$ is a degree 1 normal map and $\tau M$ is stably isomorphic to $h^{*}\left(\tau S^{4 k} \oplus \partial \gamma\right)$. So if $L(p)$ is the Hirzebruch polynomial in the Pontrjagin class $p$ of $[3,6.5]$, then

$$
\text { Sign } M=2^{2 k} L(p(\tau M))[M]=2^{2 k} L(p(\partial \gamma))\left[S^{4 k}\right] \text {. }
$$

Thus $s_{0}^{\prime}(f(\partial \gamma))=2^{2 k-3} L(p(\partial \gamma))\left[S^{4 k}\right]$. Since $\pi_{4 k-1} S O(2 d) \approx \mathbf{Z}$, we have for any $\xi \in \pi_{4 k-1} S O(2 d)$,

$$
s_{0}^{\prime}(f(\xi))=2^{2 k-3} L(p(\xi))\left[S^{4 k}\right] .
$$

Now the coefficient of the $k$ th Pontrjagin class $p_{k}(\xi)$ in $2^{2 k} L(p(\xi))$ is

$$
2^{2 k}\left(2^{2 k-1}-1\right) B_{k} /(2 k) !,
$$

where $B_{k}$ is the $k$ th Bernoulli number as in [14, Appendix B]. If $\xi$ comes from $\pi_{4 k-1} U(d)$, then $p_{k}(\xi)=(-1)^{k} \cdot 2 c_{2 k}(\xi)$. Letting $c_{2 k}^{\prime}(\xi)=c_{2 k}(\xi)\left[S^{4 k}\right] /(2 k-1)$ ! as in $\S 3$, we have

LEMMA 5.7. If $\xi \in \pi_{4 k-1} U(d)$, then

$$
s_{0}(f(\xi))=(-1)^{k} 2^{2 k-3}\left(2^{2 k-1}-1\right) B_{k} c_{2 k}^{\prime}(\xi) / k .
$$

The homomorphism $i_{*}$ of 5.6 or 5.1 restricts to $\varphi$ : image $J(u) \rightarrow$ image $J$. We shall now use this and the invariants of (a) and (c) of 5.5 to estimate image $J(u)$ in terms of image $J$. Recall that $\pi_{4 k-1} C(u) \approx \bigoplus_{j \in A} \pi_{4 k-1} U\left(d_{j}\right)$, where $d_{j}$ is the multiplicity of the eigenvalue $\lambda^{j}$ in $u$. Since the invariants $\mu$ and $s_{0}$ vanish on the torsion subgroup of $\pi_{4 k-1} C(u)$, let us treat the case when $\pi_{4 k-1} C(u)$ is free abelian, i.e. each nonzero $d_{j} \geqslant 2 k$. Assume also that $d=\sum_{j \in A} d_{j}>2 k$ so $\pi_{4 k-1} S G(2 d) \approx \pi_{4 k-1}^{s}$ is stable.

LEMMA 5.8. If $d>2 k$, the kernel of $\varphi$ is a q-group. If, in addition, each nonzero $d_{j}$ is $\geqslant 2 k$, then $\varphi$ is surjective if $k$ is odd and has cokernel $\mathbf{Z}_{2}$ if $k$ is even.

Proof. The kernel $\varphi$ is a subgroup of the kernel of $i_{*}$, which, by 5.1, is a $q$-group. The cokernel of $\varphi$ is the same as the cokernel of $\pi_{4 k-1} U \rightarrow \pi_{4 k-1} S O$ which is well known to be $\mathbf{Z}_{2}$ if $k$ is even and 0 if $k$ is odd. 
We now use $\bar{\mu}$ and $\bar{\mu}-8 \bar{s}_{0}$ to study $\operatorname{ker} \varphi$. Unfortunately, we must make the assumption that only one $d_{j}$, say $d_{1}$, is nonzero. Thus our representation $u=d \chi$. The need for this restriction is explained after 5.10 below. According to 3.6, if $\xi \in \pi_{4 k-1} C(d \chi)$, then

$$
\mu(f(\xi))=2^{2 k+1} \chi(1+\chi)^{-2 k} Q_{2 k}\left(\chi^{2}\right) c_{2 k}^{\prime}(\xi)(1-\chi)^{-2 k} .
$$

Now $1+\chi$ is a unit of $R_{\hat{G}}\left[25\right.$, p. 205] and $(1-\chi)^{-2 k}$ generates $I_{\hat{G}}^{-2 k}$, so $\mu \circ f$ $\bmod R_{\hat{G}}$ is determined by $Q_{2 k}\left(\chi^{2}\right)$. Lemma 3.5 shows that $Q_{2 k}\left(\chi^{2}\right) \in R_{\hat{G}}$ and that as long as $(2 k-1)$ ! is relatively prime to $q$ (e.g. $q \geqslant 2 k+1$ is a prime), then $\varepsilon^{\prime}\left(Q_{2 k}\left(\chi^{2}\right)\right)=Q_{2 k}(1) \neq 0 \bmod q\left(\varepsilon^{\prime}: R_{\hat{G}} \rightarrow \mathbf{Z}_{q}\right.$ the augmentation $)$, so $Q_{2 k}\left(\chi^{2}\right) \notin I_{\hat{G}}$

THEOREM 5.9. Let $q \geqslant 2 k+1$ be a prime, $k \geqslant 2$, and suppose $d>2 k$. Then the image of $J(d \chi)$ contains a subgroup of order $q$. It lies in the kernel of $\varphi$ if $q \neq 2 k+1$.

Proof. If $\xi$ generates $\pi_{4 k-1} U(d)$, the order of $\mu(f(\xi)) \bmod R_{\hat{G}}$ equals the order of $Q_{2 k}\left(\chi^{2}\right)(1-\chi)^{-2 k} \bmod R_{\hat{G}}$. $\left(I_{\hat{G}}^{-2 k} / R_{\hat{G}}\right.$ is a $q$-group.) Since $Q_{2 k}\left(\chi^{2}\right) \notin I_{\hat{G}}$ this is at least $q$. By Lemma 5.10 below, $q \in I_{\hat{G}}^{q-1} \subset I_{\hat{G}}^{2 k}$, so the order is exactly $q$. Thus $\bar{\mu}$ detects a subgroup of order $q$ in image $J(d \chi)$. For $q>2 k+1$, it lies in $\operatorname{ker} \varphi$ because image $J$ has no $q$-torsion [1].

LEMMA 5.10. If $q$ is a prime, then $q$ factors in $R_{\hat{G}}$ as $q=\theta(1-\chi)^{q-1}$ where $\theta=(1+\chi)\left(1+\chi+\chi^{2}\right) \cdots\left(1+\chi+\cdots+\chi^{q-2}\right)$. Therefore $q \in I_{\hat{G}}^{q-1}-I_{\hat{G}}^{q}$.

Proof. Let $\mathbf{Z} \hat{G} \rightarrow \mathbf{Z}[\lambda]$ be given by $\chi \mapsto \lambda$. Since $\lambda$ is a root of the polynomial $p(t)=t^{q-1}+t^{q-2}+\cdots+t+1$, our map factors through $R_{\hat{G}}$ as $E: R_{\hat{G}} \rightarrow \mathbf{Z}[\lambda]$. Because $q$ is a prime, $p$ is the minimal polynomial of $\lambda$ and $E$ is an isomorphism. The roots of $p$ are the primitive $q$ th roots of unity so $p(t)=\prod_{i=1}^{q-1}\left(t-\lambda^{i}\right)$ and $q=p(1)$ $=\prod_{i=1}^{q-1}\left(1-\lambda^{i}\right)$. Hence, in $R_{\hat{G}}, q=\Pi\left(1-\chi^{i}\right)=\theta(1-\chi)^{q-1}$. Thus $q \in I_{\hat{G}}^{q-1}$. To see that $q \notin I_{\hat{G}}^{q}$, note that $\varepsilon^{\prime}(\theta)=(q-2) ! \equiv-(q-1) \equiv+1 \bmod q$ by Wilson's theorem.

REMARK. Here is the difficulty one encounters in generalizing 5.9 to representations like that in 5.8. We find that $E(\mu(f(\xi)))=2^{2 k+1} \cdot \sum_{j \in A} c_{2 k}^{\prime}\left(\xi_{j}\right) e_{j}$ where

$$
2 e_{j}=2 \lambda^{j}\left(1+\lambda^{j}\right)^{-2 k} Q_{2 k}\left(\lambda^{2 j}\right)\left(1-\lambda^{j}\right)^{-2 k}=(2 k-1) ! \Phi_{2 k}\left(\lambda^{j}\right) .
$$

As in 5.9, the $e_{j}$ satisfy $q e_{j} \in \mathbf{Z}[\lambda]$ but $e_{j} \notin \mathbf{Z}[\lambda]$. Moreover, $\left\{e_{j} \mid j \in A\right\}$ is a basis for $\mathbf{Q}\left(\lambda+\lambda^{-1}\right)$ over $\mathbf{Q}$ since $\left\{\Phi_{2 k}\left(\lambda^{j}\right)\right\}$ is [7]. One would like to conclude that $E(\mu(f(\xi)))$ $\bmod \mathbf{Z}[\lambda]$ generates a $\mathbf{Z}_{q}$ vector space of dimension equal to the rank of $\pi_{4 k-1} C(u)$ as $\xi$ runs through $\pi_{4 k-1} C(u)$. Unfortunately, this does not follow from the rational linear independence of the $e_{j}$. Perhaps it can be proved if we know more about $\Phi_{2 k}\left(\lambda^{j}\right)$.

We have not yet used the invariant $\bar{\mu}-8 \bar{s}_{0}$ which, on the face of it, is stronger than $\bar{\mu}$. But if $q>2 k+1, q \in I_{\hat{G}}^{q-1} \subset I_{\hat{G}}^{2 k+2}$, so $\mu(f(q \cdot \xi)) \in I_{\hat{G}}^{2}$ for $\xi \in \pi_{4 k-1} C(u)$. Also, our formula for $s_{0}(f(\xi))$ preceding 5.7 shows that $s_{0}(f(q \cdot \xi)) \in I_{\hat{G}}^{q-1}$ if $q>2 k+1$ since the denominator of $B_{k} / k$ is prime to $q$. Thus $\bar{\mu}-8 \bar{s}_{0}$ is no better than $\bar{\mu}$. However, if $q=2 k+1$, the story is different. In that case, $\bar{\mu}$ detects only the $q$-torsion which $\varphi$ takes to image $J$. 
THEOREM 5.11. Let $q=2 k+1$ be a prime and $d>2 k \geqslant 4$. Then the image of $J(d \chi)$ contains a cyclic subgroup of order $q^{2}$ whose subgroup of order $q$ lies in $\operatorname{ker} \varphi$.

Proof. Let $e=\chi(1+\chi)^{-2 k} Q_{2 k}\left(\chi^{2}\right)(1-\chi)^{-2 k}$ and $\beta=(-1)^{k} B_{k}\left(2^{2 k-1}-1\right) / k$. Then $\left(\mu-8 s_{0}\right)(f(\xi))=2^{2 k}(2 e-\beta) \cdot c_{2 k}^{\prime}(\xi)$. We shall show that $q \cdot(2 e-\beta) Z$ $0 \bmod I_{\hat{G}}^{2}$ but that $q^{2}(2 e-\beta) \equiv 0 \bmod I_{\hat{G}}^{2}$. This will prove that the image of $(\bar{\mu}-$ $\left.8 \bar{s}_{0}\right) \circ f$ is cyclic of order $q^{2}$. The remark about the subgroup of $\operatorname{ker} \varphi$ follows since image $J$ has $q$-torsion cyclic of order $q$ which must come from $\pi_{4 k-1} C(d \chi)$ by 5.8 .

Observe that any real element of $I_{\hat{G}}$ is automatically in $I_{\hat{G}}^{2}$. Since $\mu-8 s_{0}$ is real valued, this allows us to work $\bmod I_{\hat{G}}$ instead of $\bmod I_{\hat{G}}^{2}$. The advantage of this is that elements of $R_{\hat{G}}$ are congruent $\bmod I_{\hat{G}}$ to their augmentations.

Now we reduce $2 q e$ and $q \beta \bmod I_{\hat{G}}$. Using 3.5 and 5.10,

$$
\begin{aligned}
2 q e & \equiv 2 \cdot 2^{-2 k} Q_{2 k}(1) q \cdot(1-\chi)^{-2 k} \\
& \equiv 2 \cdot 2^{-2 k}(-2)^{2 k-1}(2 k-1) ! \theta \\
& \equiv-(q-2) ! \equiv-1 \bmod I_{\hat{G}}
\end{aligned}
$$

Next, according to [14, B.3], $(-1)^{k} q B_{k} \equiv+1 \bmod q$. The remaining factor of $\beta$ :

$$
\left(2^{2 k-1}-1\right) / k \equiv\left(2^{q-2}-1\right) / k \equiv-1 / 2 k \equiv+1 \quad \bmod q .
$$

Thus $q \beta \equiv+1 \bmod I_{\hat{G}}$ and $2 q e-q \beta \equiv-2 \bmod I_{\hat{G}}$. The theorem follows.

EXAMPLE 5.12. Let $k=2$ or $3, d>2 k$. Then $J: \pi_{4 k-1} S O(2 d) \rightarrow \pi_{4 k-1}^{s}$ is onto. Using this and a Postnikov decomposition of $L^{2 d-1}$ one can show that if $q>2 k+1$

$$
\pi_{4 k-1} F(d \chi) \approx \mathbf{Z}_{q} \oplus \pi_{4 k-1}^{s}
$$

and if $q=2 k+1$ we have an exact sequence

$$
0 \rightarrow \mathbf{Z}_{q} \rightarrow \pi_{4 k-1} F(d \chi) \rightarrow \pi_{4 k-1}^{s} \rightarrow 0
$$

and the $q$-torsion in $\pi_{4 k-1} F(d \chi)$ is $\mathbf{Z}_{q^{2}}$.

Therefore our invariants $\bar{\mu}$ and $\bar{\mu}-8 \bar{s}_{0}$ detect all the $q$-torsion in image $J(d \chi)$. It would be interesting to know if they always do this.

Using 5.8 and 5.1 one may show that when $k=2, J(d \chi)$ has cokernel $\mathbf{Z}_{2}$. Therefore $R S_{7 \oplus d \chi} \neq$ image $f+$ image $g$ (see the third paragraph after 5.4). In fact, there are even smooth $7 \oplus d \chi$-spheres which are not obtained from homotopy smoothings of $D^{8} \times L^{2 d-1}$ or $\pi_{7} C(d \chi)$.

Finally, $\pi_{8} F(d \chi) \approx \mathbf{Z}_{2} \oplus \mathbf{Z}_{2}$. Thus 5.3 and the result $\pi_{8} F(d \chi) \approx$ Tor $\pi_{8}(F(d \chi), C(d \chi))$ yield

$$
R S_{8 \oplus d \chi} \approx \mathbf{Z}_{2} \oplus \mathbf{Z}_{2}, \quad C S_{8 \oplus d \chi} \approx \mathbf{Z}_{2} \oplus \mathbf{Z}_{2} \oplus \Gamma_{8} \approx \mathbf{Z}_{2} \oplus \mathbf{Z}_{2} \oplus \mathbf{Z}_{2} .
$$

\section{REFERENCES}

1. J. F. Adams, On the groups $J(X)$. IV, Topology 5 (1966), 21-71.

2. M. F. Atiyah and R. Bott, A Lefschetz fixed point formula for elliptic complexes. II, Ann. of Math. (2) 88 (1968), 451-491.

3. M. F. Atiyah and I. M. Singer, The index of elliptic operators. III, Ann. of Math. (2) 87 (1968), 546-604.

4. A. Bak, Odd dimension surgery groups of odd torsion groups vanish, Topology 14 (1975), 367-374

5. W. Browder and T. Petrie, Diffeomorphisms of manifolds and semifree actions on homotopy spheres, Bull. Amer. Math. Soc. 77 (1971), 160-163. 
6. P. Conner, Differentiable periodic maps, Lecture Notes in Math., vol. 738, Springer-Verlag, Berlin and New York, 1979.

7. J. Ewing, Spheres as fixed point sets, Quart. J. Math. Oxford Ser. (2) 27 (1976), 445-455.

8. $431-442$. , Semifree actions of finite groups on homotopy spheres, Trans. Amer. Math. Soc. 245 (1978),

9. D. Husemoller, Fibre bundles, Graduate Texts in Math., Vol. 20, Springer-Verlag, Berlin and New York, 1966.

10. M. A. Kervaire and J. W. Milnor, Bernoulli numbers, homotopy groups, and a theorem of Rohlin, Proc. Internat. Congr. Math., Edinburgh, Cambridge Univ. Press, Cambridge, 1958, pp. 454-458.

11. R. Lashof and M. Rothenberg, G-smoothing theory, Proc. Sympos. Pure Math., vol 32, Amer. Math. Soc., Providence, R.I., 1978, pp. 211-266.

12. J. P. May, The homotopical foundations of algebraic topology, Mimeo, Univ. of Chicago.

13. J. W. Milnor, Whitehead torsion, Bull. Amer. Math. Soc. 72 (1966), 358-426.

14. J. W. Milnor and J. D. Stasheff, Characteristic classes, Ann. of Math. Studies, No. 76, Princeton Univ. Press, Princeton, N.J., 1974.

15. M. Rothenberg, Torsion invariants and finite transformation groups, Proc. Sympos. Pure Math., vol. 32, Amer. Math. Soc., Providence, R.I., 1978, pp. 267-311.

16. Differentiable group actions on spheres, Proc. Advanced Study Inst. Algebraic Topology, Aarhus, Matematisk Institut, Aarhus, 1970.

17. M. Rothenberg and J. Sondow, Nonlinear smooth representations of compact Lie groups, preprint.

18. R. Schultz, Homotopy sphere pairs admitting semifree differentiable actions, Amer. J. Math. 96 (1974), 308-323.

19. __ Smooth actions of small groups on exotic spheres, Proc. Sympos. Pure Math., vol. 32, Amer. Math. Soc., Providence, R.I., 1978, pp. 155-160. , Differentiable group actions on homotopy spheres. I, Invent. Math. 31 (1975), 105-128.

21. Differentiable group actions on homotopy spheres. II, preprint.

22. J. Shaneson, Wall's surgery obstruction groups for $\mathbf{Z} \times G$, Ann. of Math. (2) 90 (1969), 296-334.

23. E. Spanier, Algebraic topology, McGraw-Hill, New York, 1966.

24. C. T. C. Wall, Non-additivity of the signature, Invent. Math. 7 (1969), 269-274.

25. __ Surgery on compact manifolds, Academic Press, New York, 1970.

26. L. Smith, The e invariant and finite coverings, Indiana Univ. Math. J. 24 (1975), 659-675.

27. R. Stong, Notes on cobordism theory, Princeton Univ. Press, Princeton, N.J., 1968.

School of Mathematics, Institute for Advanced Study, Princeton, New Jersey 08540

Current address: Department of Mathematics, The Ohio State University, Columbus, Ohio 43210 